Article

\title{
Detecting Vasodilation as Potential Diagnostic Biomarker in Breast Cancer Using Deep Learning-Driven Thermomics
}

\author{
Bardia Yousefi $^{1, *\left(\mathbb{D}, \text { Hamed Akbari }^{2} \text { and Xavier P.V. Maldague }\right.}{ }^{1, *}$ \\ 1 Department of Electrical and Computer Engineering, Laval University, Quebec City, QC G1V 0A6, Canada \\ 2 Department of Radiology, University of Pennsylvania, Philadelphia, PA 19104, USA; AkbariHA@upenn.edu \\ * Correspondence: Bardia.Yousefi.1@ulaval.ca (B.Y.); Xavier.Maldague@gel.ulaval.ca (X.P.V.M.)
}

Received: 23 September 2020; Accepted: 30 October 2020; Published: 31 October 2020

check for updates

\begin{abstract}
Breast cancer is the most common cancer in women. Early diagnosis improves outcome and survival, which is the cornerstone of breast cancer treatment. Thermography has been utilized as a complementary diagnostic technique in breast cancer detection. Artificial intelligence (AI) has the capacity to capture and analyze the entire concealed information in thermography. In this study, we propose a method to potentially detect the immunohistochemical response to breast cancer by finding thermal heterogeneous patterns in the targeted area. In this study for breast cancer screening 208 subjects participated and normal and abnormal (diagnosed by mammography or clinical diagnosis) conditions were analyzed. High-dimensional deep thermomic features were extracted from the ResNet-50 pre-trained model from low-rank thermal matrix approximation using sparse principal component analysis. Then, a sparse deep autoencoder designed and trained for such data decreases the dimensionality to 16 latent space thermomic features. A random forest model was used to classify the participants. The proposed method preserves thermal heterogeneity, which leads to successful classification between normal and abnormal subjects with an accuracy of $78.16 \%(73.3-81.07 \%)$. By non-invasively capturing a thermal map of the entire tumor, the proposed method can assist in screening and diagnosing this malignancy. These thermal signatures may preoperatively stratify the patients for personalized treatment planning and potentially monitor the patients during treatment.
\end{abstract}

Keywords: vasodilator activity; breast cancer screening; imaging biomarker; deep sparse autoencoder; dimensionality reduction; deep-learning features

\section{Introduction}

Breast cancers caused an estimated 41,760 deaths out of 606,808 overall deaths for females and 500 deaths for males, while the estimated new cases were 271,270 deaths for both genders (268,600 women and 2670 men) in the United States in 2019. This evidence shows that despite considerable advancement in breast cancer screening and treatment, breast cancer is still the second cause of cancer death among women [1]. Clinical breast exam (CBE), magnetic resonance imaging (MRI), mammography, and ultrasound are widely used for the diagnosis of breast cancer. Among them, CBE and mammography are considered the most common breast screening tools [1]. This study proposes machine learning techniques and analyses using infrared thermography as a new technique for breast cancer screening. We hypothesized that thermal heterogeneity may associate with angiogenesis, nitric oxide vasodilatory phenomena, inflammation, and estrogen caused by cancer symptoms.

Mammography has been the gold standard for diagnosing breast cancer since the early 1960s despite numerous studies indicating the variability of this imaging modality is affected by breast density, 
age, type of problem, and family history [2-5]. Mammography showed weakness in being used for breast cancer screening for women with dense breasts, hormone replacement therapy, and fibrocystic breasts [6-9]. Research showed the detection rate of mammography considerably diminishes by increasing breast density among the patients [7]. The studies show that age has a reverse association with breast cancer detection using mammography [10]. Moreover, findings show that during mammography the pressure on the breast tumors is adequate to rupture the encapsulated tumors (depends on the location of the tumor) and possibly circulate malignant cells in the bloodstream [11].

The risk of radiation using mammography is one of the important disadvantages of using this imaging modality. Younger women are more susceptible to the risk of radiation-induced breast cancer than older women due to their undifferentiated cells being prone to influence by ionizing radiation [5,10-15].

Family history of breast cancer and/or the $B R C A 1 / 2$ gene mutations are other factors that this gene mutation may be the result of radiation effect, which might be induced indirect effects to damage DNA by producing reactive oxygen species (ROS) from the cell's water molecules [16,17]. Some studies showed that the ionizing radiation by mammography might be more dangerous for patients with such mutations [16-19].

For the dense breast, ultrasound can be an adjunctive tool used with mammography screening for detecting the abnormality [2]. However, it shows a dependency on tumor size, palpability, breast density, tools' quality, physician's expertise who performs the procedure, and interpreting the image [2,5,20-22]. Magnetic resonance imaging (MRI) is also an alternative imaging modality, which can identify early breast cancer in the place where conventional imaging fails to detect the abnormalities [23,24]. Ahen et al. (2014) concluded that the high costs and low specificity of MRI limits the popularity of MRI for annual screening for high-risk women [25]. CBE is considered as a great alternative conducted by the clinician and can help to detect at least $50 \%$ of asymptomatic breast cancers but has not been used alone [26,27].

\section{Thermography and Biological Rationale as an Alternative Imaging Modality}

Infrared thermography is used as an additional cost-effective alternative for breast cancer screening as a non-invasive procedure that does not pressurize the breast tissue nor expose the body to ionizing radiation. The skin emission is about 0.98 , which is close to the emission of the blackbody. Thermal radiation emitted from the body has a wavelength of $8-10 \mu \mathrm{m}$ bandwidth, which can be captured by the infrared camera [28-30]. Due to the relatively lower sensitivity of thermographic screening, it usually adds to other diagnosing methods, mainly with CBE to increase the overall diagnostic accuracy [31,32].

Blood circulation is the main contributor to heat transfer in the body. Vascularity is also considered an important parameter for heat transfer [33]. Evidence supports different thermal conductivity between normal tissues in breasts and cancerous lesion thermal profile discrepancy [34], also abnormal skin temperature manifestation is an indicator of pathological changes explained by metabolic activity associated with the tumor such as angiogenesis, nitric oxide, inflammation, and estrogen [5,33,35]. Changing in the endocrine due to the presence of tumors alters the thermal profile by changing the vascularization of the tissues to deliver oxygen and nutrients to tumors [36], in the process of pathologic angiogenesis. In such chaotic and pathological processes of angiogenesis, smooth muscle cells receiving abnormal vasoconstrict blood vessels in the area [36]. Several studies proved the value of infrared thermography on detecting hypervascularity and hyperthermia on non-palpable breast cancer [36-38].

Tumor angiogenesis and metastatic behavior are biomarkers of breast carcinoma and c-Met pathway activation, which are used also for tumor progression. Also, there is an association of c-Met and downstream signaling pathways with angiogenesis that can be assessed by microvessel density (MVD) [39]. Infrared was used to assess the existence of MVD, which is associated with endothelial cell (CD34) marker and downstream signaling pathways (angiogenesis, RAS-MAPK, and PI3K-AKT) [39]. 
In another study, there is a relationship between MVD and tumor-associated macrophages (TAMs) and vascular endothelial growth factor (VEGF) expression [23,40,41]. In addition, white blood cells produce nitric oxide as a defense mechanism against cancerous cells that is a vasodilatory substance [31,37]. Nitric oxide performs as a vasodilator in cancerous tissues in the breasts to enhance oxygen and nutrient delivery, which increases the local temperature in the area [42-44]. In general, cancer (including breast cancer) is influenced by different cellular factors including reactive oxygen and nitrogen species (RONS) and, as reported, hypoxic condition elevated RONS production [44-47]. Nitric oxide has reactive diatomic free radical plays a role in promoting and inhibiting cancer [48].

DNA damage occurs once nitric oxide reacts to form other reactive metabolites as well as nitrite, peroxynitrite, nitrate, or S-nitroso-thiols, which provoke genotoxic effects $[48,49]$. Reactive nitrogen species (RNS) exposure causes post-translational changes, which leads to different interactions by other cellular targets and causes diverse locally dependent concentration effects $[49,50]$. In the late stages of breast cancer also, c-Met promotes metastases by having vascular reprogramming and inflammatory cytokine upregulation [51], inflammation-related cytokine tumor necrosis factor-alpha $(\mathrm{TNF}-\alpha)$ in tumor invasion [52]. This often happens due to a long period of remission before the diagnosis of breast cancer [53], which can be detected faster using the combination of CBE and infrared screening. The presence of inflammation is another mechanism of local heat generation. Cancer causes a vasodilatory response, due to the inflammatory cell involvement' which increases temperature [42]. Estrogen also facilitates vasodilation by locally enhancing nitric oxide production. Imbalanced estrogen could change the vasodilatory effects of the tissue resulting in thermal variations [54]. Evidence shows that ecto- $5^{\prime}$ - nucleotidase $(\mathrm{eN})$ is negatively controlled by estrogen receptor- $\alpha(\mathrm{ER} \alpha)$. This suggests that eN expression and its adenosine generation associate with breast cancer progression. eN expression in estrogen receptor-negative cells considered to be an aggressive breast cancer biomarker [55].

Such process metabolic heat generation investigated for normal and cancerous breast tissues and its rates reached $20 \mathrm{~K} \mathrm{~W} / \mathrm{m}^{3}$ and the range between $100 \mathrm{~K}-1200 \mathrm{~K} \mathrm{~W} / \mathrm{m}^{3}$ for two types of tissues, respectively [56]. Despite United States Food and Drug Administration (FDA) approval for using infrared thermography, it can be used as an adjunct screening modality along with MRI, mammography, and ultrasound $[57,58]$. A visual summary of the factors influencing the heterogeneity in thermal imaging are presented in Figure 1.
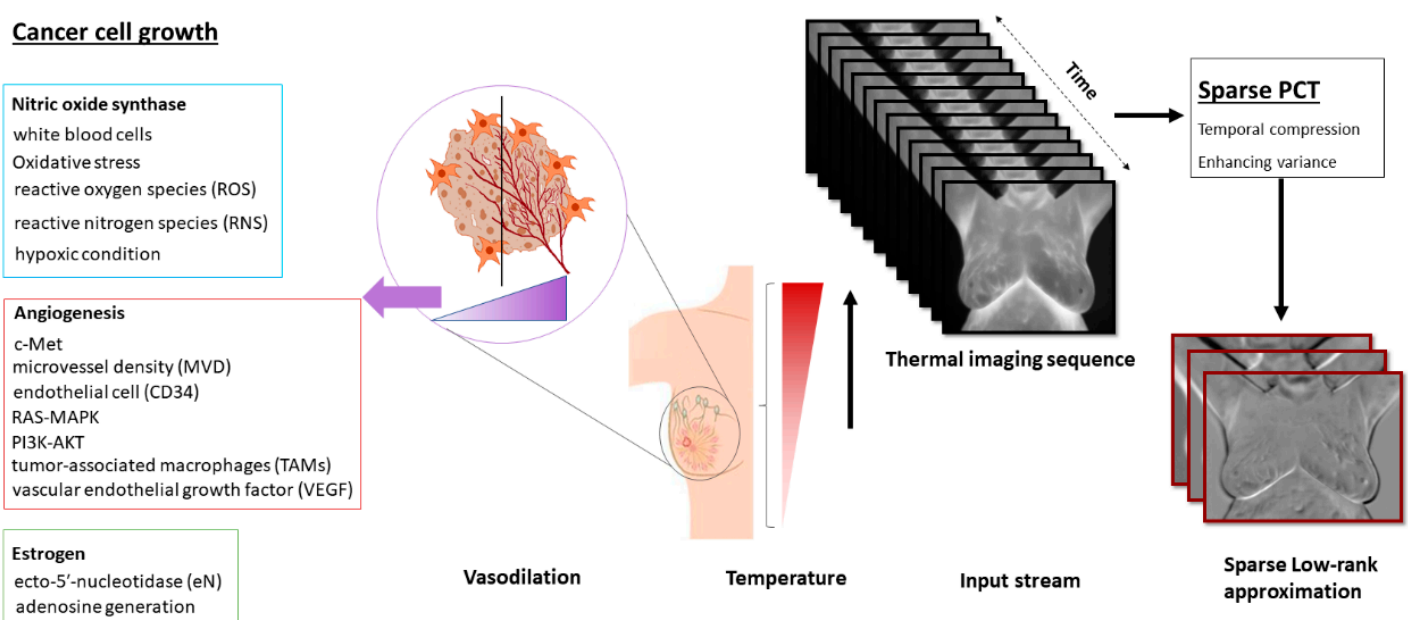

Figure 1. The block diagram of the biological connection to the response of infrared thermography as a fast step with other methods such as clinical breast exam (CBE) in breast cancer screening and cancer presence in the breast area are shown.

In this study, we propose a method to use high-dimensional deep-learning features to track the vasodilator activities in the breast area as a potential biomarker in detecting breast cancer. The contributions of the paper are as follows: 
- The sparse principal component analysis in the thermography (Sparse PCT) is used to compress the input thermal sequence and capture high temporal variance across the acquisitions. This leads to capture thermal heterogeneity patterns in three first initial bases, called avatars, which concatenate in three different channels similar to a red, green and blue (RGB) image as the input for our pretrained model.

- Deep thermal features, called deep-thermomics inspired by radiomics, are extracted to measure thermal heterogeneity in breast cancer screening using infrared thermography.

- The proposed approach tackles the problem of the curse of dimensionality in deep-thermomics using a sparse deep autoencoder without using traditional human-engineered feature selection methods.

- The multivariate models trained and validated using the obtained descriptors successfully classify between symptomatic and non-symptomatic subjects. We also provided a comparative analysis using a non-sparse PCT.

- This study shows the association between thermal heterogeneous patterns and potential vasodilation in the breast area, as a new potential imaging biomarker.

The rest of the paper is organized as follows. In the next section, thermal transfer in passive thermography is summarized. In Section 3, the methodology of the approach will be briefly described by applying sparse PCT analysis for thermography and pre-trained ResNet-50 deep neural networks. The experimental results are presented in Section 5, and the discussion is in Section 6. The conclusions are summarized in Section 7.

\section{Thermal Transfer in Thermography}

A thermal camera captures the spatial heterogeneity of temperature on the targeted region of interest (ROI) over time. This heat transient can be through active or passive thermography techniques. In general, the thermal transfer/heat conduction equation of a specimen can be summarized by the following equation:

$$
\rho C_{p} \frac{\partial T}{\partial t}=k \frac{\partial^{2} T}{\partial t^{2}}+\dot{q}
$$

where $T=T(x, y, z)$ is a temperature field, $k$ is thermal conductivity constant from the material $(\mathrm{W} / \mathrm{m} . \mathrm{K}) . \rho$ is the density $\left(\mathrm{kg} / \mathrm{m}^{3}\right), C_{p}$ is specific heat $(\mathrm{J} / \mathrm{kg} \cdot \mathrm{K}), \dot{q}(x, y, z, t)$ is the internal heat generation function per unit volume, in the passive thermography.

Applying infrared thermography on biological organs and tissues, as a complex structure, composed of fat, blood vessels, parenchymal tissues, and nerves with some uncertainty for the rate of blood perfusion and metabolic activity. Pennes' bioheat equation [59] provides accurate thermal computations and states as follows:

$$
\rho_{t} c_{t}\left(\frac{\partial T_{t}}{\partial t}\right)=\nabla \cdot\left(k_{t} \nabla T_{t}\right)+\omega_{b} c_{b}\left(T_{a}-T_{t}\right)+q_{m}
$$

where $\omega_{b}$ represents the flow rate of blood, $q_{m}$ is the metabolic rate (heat generation), and $b$, and $a$ in $\omega_{b} c_{b}\left(T_{a}-T\right)$ the additive term stands for blood, and arteries (in targeted tissue), respectively.

\section{Methodology}

Infrared thermography records thermal heterogeneity in the subdermal area of the breast in temporal order. To capture such effect by abstracting such patterns, low-rank matrix approximation is used to maximize the variance across thermal acquisition time.

\subsection{Low-Rank Approximation of Thermal Stream}

Low-rank matrix approximation is commonly used in thermography [60-68], due to capturing thermal variations across the temporal order in the sequence. This leads to detecting thermal patterns on the subsurface of specimens. Such analyses capture thermal heterogeneity in the skin area for breast 
cancer screening patients. Principal component analysis (PCA), called PCT for thermography [60], through singular value decomposition (SVD) used for decomposing the input matrix (heat matrix) $X$, which is $p \times n$, where $n$ is the vectorized thermal image (breast screening) in every sequence and $p$ corresponds to the number of observations and decomposes to:

$$
X=U \Gamma V^{T}
$$

where $\mathrm{U}$ is the $p \times n$ matrix $(p>n)$ and $\Gamma$ is a diagonal matrix with a dimension of $n \times n$ and either zero or positive elements. $V^{T}$ denotes the transpose of the $n \times n$ matrix. The method captures the spatiotemporal variance by selecting the bases correspond to $80 \%$ of variance from the eigenvector matrix. Matrix U represents the bases of the input matrix.

The PCT is a linear transformation technique that decomposes the input zero-mean data matrix into the bases and coefficient matrix. To find the optimal solution for such transformation, $\ell_{2}$ and $\ell_{1}$ penalty terms with regularization parameters were added to the PCT, which led to Sparse PCT [61,62] and increased the performance of such a technique, particularly when encountering additive noise.

Such modifications in Sparse PCT not only turned PCT into a nonlinear transformation but following the same maximization of the variance among the bases. If the empirical covariance matrix of $X_{p \times n}$ is presented by $X^{T} X$, Sparse PCT is the maximization of variance in the direction of vector $v \in \mathbb{R}^{p}$ for $1 \geq k \geq p$.

$$
\begin{gathered}
\max v^{T} \Sigma v \\
\text { such that }\|v\|_{2}=1,\|v\|_{0} \geq k
\end{gathered}
$$

Let $v$ be a variance of the input matrix and $\|v\|_{0}$ be $\ell_{0}$ norm of $v$, which is the non-zero components. This is an NP-hard (non-deterministic polynomial-time hard) problem and Zou et al. (2006) and elastic net algorithms used to solve this optimization [69]. Sparse PCT showed considerable performance in thermography to find a low-rank approximation of input thermal images. Here, we applied sparse PCT to preserve thermal heterogeneity in the subsurface of skin as a potential biomarker leading to early diagnosis of breast cancer.

Let $I$ is thermal imaging stream taken from the participants such that $I \in \mathbb{R}^{n \times m \times \tau}$. If $x$ is a vectorized matrix named heat matrix made by stacking vectorized infrared images, $x \in \mathbb{R}^{n \cdot m \times k}$. $B=\left\{\beta_{1}, \beta_{2}, \ldots, \beta_{\tau}\right\}$ denotes a set of bases obtained by sparse PCT. Each $\beta$ cropped to a squared matrix focusing more on the ROI as an input to the ResNet-50 (spatial dimension of $224 \times 224$ ). Using $k=3$ corresponding to three predominant low-rank matrix approximation, we capture dynamic variations on thermal images in the ROI during $\tau$ time.

\subsection{Deep Thermomics}

Deep neural networks and particularly convolutional neural networks (CNN) are widely used by researchers in various fields with diverse applications, comprising image processing, and particularly medical imaging. $\mathrm{CNN}$ is a group of connected deep neural networks that uses a variation of multi-layer perceptron with many hidden layers [70]. The hidden layers of CNN normally consist of convolutional (cross-correlation) layers (filtering), pooling layers, rectifier layer (ReLu), fully connected layers, and normalization layers [71,72]. Several adaptive filters (as kernels) with small receptive fields layers makes CNN different from other similar deep neural networks. Because of such filtering in the input layers using a 2-dimensional dot product between the filter entries and the input data, the model extracts some features with higher sensitivity in spatial positions of input. This increases the applications of CNN-based networks in a variety of applications with the focus of imaging. Some of these networks are already trained for specific imaging datasets and used as a pre-trained network to perform classification or recognition.

After the success of the AlexNet [72] in image processing at the LSVRC2012, deep residual network (ResNet) [73] was perhaps the most innovative research in the computer vision and deep learning 
research community. ResNet provides the ability of a trainable network with many layers while holding a compelling performance.

The state-of-the-art CNN architectures are going deeper such as the very deep $\mathrm{CNN}$ for large-scale visual recognition (VGG) network [74], GoogleNet (also codenamed Inception-v1) [75] that have 19 and 22 layers respectively. ResNet tackle the vanishing gradient issue by introducing an "identity shortcut connection" that skips one or more layers [73], which does not degrade the network performance, since it simply stacks identity mappings in every layer. The pre-activation variant of residual block [76] increases the popularity of ResNet with an excessive number of hidden layers in the computer vision and medical image processing.

A deep learning method has been employed to non-invasively detect chemically treated collagenous tissue nonlinear anisotropic stress-strain responses in the microscopic images [77]. VGG16 is used for the prognosis of glioblastoma and as a radiographic biomarker for noninvasive categorization between true progression and pseudo-progression in these patients [78]. The initial application of the deep features in infrared analyses has been presented for finding defective patterns in the specimens using spectral difference among various areas of specimens [79]. Using traditional dimensionality reduction or feature selection is not a substantial way due to the low-level status of hidden weights in this model, which might be perceived as collinearity among features. In infrared breast cancer screening methods, a comparative analysis on AlexNet, GoogLeNet, ResNet-18, VGG-16, and VGG-19 for 88 patients using a pre-trained model resulted in discrimination between normal and pathologic patients [80]. ResNet50 was applied to extract features from histopathological images and followed by autoencoder, K-means clustering to choose discriminative patches using PCA to diagnose breast cancer [81]. A CNN approach tackled the same dataset using ResNet34 and ResNet50 and achieved a significant performance on detection of breast cancer in blind validation, and used the entire thermal sequences as the input of their system [82]. A cohort of 57 cases used applying a new configuration of CNN showed promising accuracy, while they outperformed ResNet50, SeResNet50 and Inception models [83]. Similarly, CNN used with additional algorithms such as with Bayes algorithm [84] or support vector machine (SVM) [85] to conduct diagnosis assessments.

In this paper, a pre-trained residual deep convolutional network for large-scale image recognition (ResNet-50) [73,86] was used. ResNet-50 is used as a hybrid feature generator (deep features). The method uses low-rank matrix approximation of thermal sequences as a sparse representation of the whole set, called avatar. Three first bases make three channels representing the entire thermal set as an input to the ResNet-50 model and extracted deep-thermomic features, 2048 size vector, as output.

Since the input of the ResNet-50 model requires an RGB squared image, we leverage this property to embed three first bases obtained by sparse PCT as three channels of the input image, showed by $\psi$, where $\psi_{R G B} \longrightarrow \psi_{\beta_{1} \beta_{2} \beta_{3}}$ (see Figure 2). Applying feed-forward convolution in neural network lookalikes of multiple-internal-functions gives:

$$
\mathcal{F}(\psi)=\mathcal{F}_{L}\left(\ldots \mathcal{F}_{2}\left(\mathcal{F}_{1}\left(\psi ;\left\{W_{1}\right\}\right) ;\left\{W_{2}\right\}\right) \ldots ;\left\{W_{L}\right\}\right) . \quad \mathcal{F}: \mathbb{R}^{224 \times 224 \times 3}
$$

Let $\mathcal{F}_{i}$ represents the residual mapping to be learned and $\left\{W_{i}\right\}$ denotes weights in each layer. The regular linear convolution involves a filter bank where the output also contains the input dimensional property. The last layer contains a vector by the size of 2048 and links to three channels low-rank representation of infrared stream. This gives 2048 low-level features from the image used as input to the deep learning-based dimensionality reduction model. 


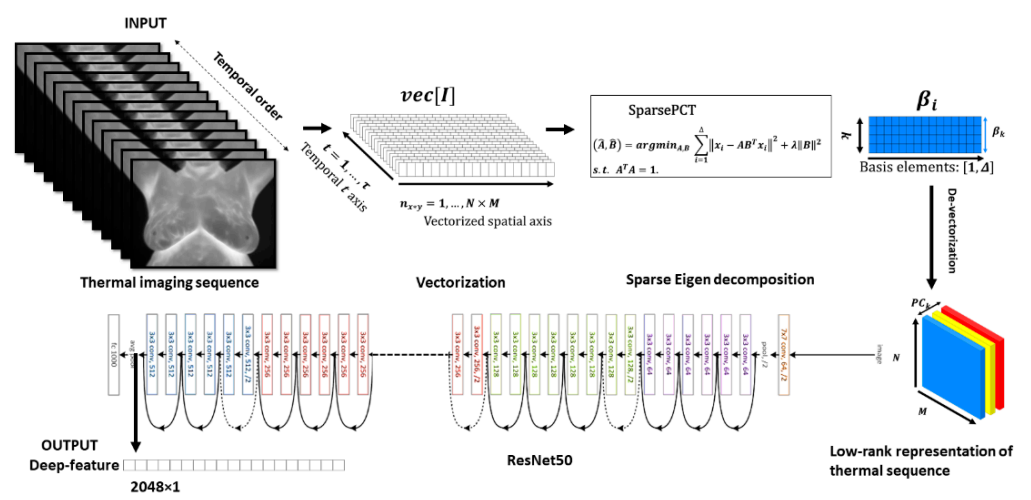

Figure 2. Workflow of the proposed approach in temporal compression and extraction of low-rank matrix approximation and generating the deep thermomics using residual network (ResNet-50) is presented.

\subsection{Sparse Autoencoder for Dimensionality Reduction for Deep Thermomics}

Radiomics, high-throughput features, refer to sub-visual/quantitative feature extraction and consider a vital part of medical image analysis and radiology which strive to exploit the amount of quantitative minable features extract from imaging data [87]. Every feature contains a distinct phenotype of the tumor which may have diagnosis/prognostic power and adjunctive clinical importance across the different diseases. In oncology, identified features from radiology or any other imaging data facilitate prediction, diagnosis, and prognosis associated with cancer disease to monitor the response, like survival, as a progression criterion of disease and treatment response. Pretrained deep neural networks provide high-dimensional features as an opportunity to gain information on tumor area and its environment that is not otherwise available to the radiologist.

Having sufficed features from the medical images leverage better diagnostic/prognostic decisions whereas high-dimensional feature space can impede computation and enervates the performance of feature selection known as the curse of dimensionality problem. This creates a wrong outcome of the model due to overfitting the decision-making unit. Traditional feature selection might not the best solution for such an issue because the low-level informative features provided by the network might translate as collinearity among the descriptors and lead to the elimination of valuable information. Here, we propose an autoencoder trained specifically for such high-dimensional throughput features to reduce the dimensionality hierarchically to the lower dimension. Autoencoders are data compression algorithms that make of hierarchical compression and decompression units cascade to each other. They are data-specific, automatically learn from training input data instead of being obtained by human interference, and lossy. Autoencoders can compress data like what they have been trained on and cannot be generalized for other dissimilar data, while they are different from lossless arithmetic compression [88]. An autoencoder contains encoding and decoding parametric functions with a measure of distance, or "loss" function, between the compressed representation of the input data and the final decompressed representation. The parameters of the encoding/decoding functions can be optimized by using stochastic gradient descent to minimize the reconstruction loss.

Autoencoder architecture. Several dense layers with different sizes were employed to reduce the dimensionality from 2048 to 16 compressed descriptors, in the latent space. Eight dense (8D) layers, including 4 dense layers in each of encoder and decoder, were used. The intermediate representation of feature dimensionality was varied from the size of 1024,256 , and then 64 , to latent space with size 16. Each layer has a ReLu activation function and the last layer has a Sigmoid activation function, with sparse constraints in the initial layer (Figure 3). The network trained for the batch size of 128, with a total of 3000 iterations, with an unfixed learning rate in the Adam optimization algorithm.

Let $x \in \mathbb{R}^{F}$ considers as the first mapped input, where $F=2048$, to the latent space with $h=f_{e}(x)=a_{e}\left(W x+b_{e}\right)$ is the hidden representation of the input vector, $a_{e}$ is the encoder activation, $W \in \mathbb{R}^{F \times G}$ is the weight matrix, and $b_{e} \in \mathbb{R}^{F}$ is the encoder bias. $y=f_{d}(h)=a_{d}\left(W^{T} h\right)$ span the latent 
features back to the original space and $y$ is the counterpart of $x$ and $a_{d}$ is the activation for the decoder. Since we use a deep autoencoder encoder and decoder functions are expanded for multilayer as $h_{i}=f_{e_{i}}\left(\ldots f_{e_{2}}\left(f_{e_{1}}(x)\right)\right)=a_{e_{i}}\left(\ldots a_{e_{2}}\left(W_{2} a_{e_{1}}\left(W_{1} x+b_{e_{1}}\right)+b_{e_{2}}\right)\right) y_{i}=f_{d_{1}}\left(\ldots\left(f_{d_{i-1}}\left(f_{d_{i}}\left(h_{i}\right)\right)\right)\right)=$ $a_{d_{1}}\left(W_{1}^{T} \ldots\left(W_{i-2}^{T} a_{d_{i-1}}\left(W_{i-1}^{T} a_{d_{i}}\left(W_{i}^{T} h_{i}\right) h_{i-1}\right)\right)\right)$, respectively. The objective of an autoencoder is to minimize $\left\{W_{i}, b_{e_{i}}\right\}$ :

$$
\mathcal{J}_{A E}=\mathbb{E}_{x}\left[\ell\left(x, f_{d_{i}}\left(f_{e_{i}}(x)\right)\right)\right]
$$

This captures the predominant patterns in the data and provides a noise invariant representation (manifold) of data which is very valuable considering the sensitivity of the infrared to noise. $\ell($.) denotes the loss function and here, we use binary cross-entropy (BCE), as presented below:

$$
\mathcal{L}_{B C E}=-\frac{1}{F} \sum_{i=1}^{F} y_{i} \log \left(p\left(y_{i}\right)\right)+\left(1-y_{i}\right) \log \left(1-p\left(y_{i}\right)\right)
$$

where $y$ is the label and $p(y)$ is the predicted probability of the segmented label for all $F$ points. Having a Sigmoid function, $\frac{1}{1+e^{-y}}$ makes the function a binarized value, representing the existing class against background class.

Learning a dictionary fitted to a training set with the sparse latent code is formulated by the optimization below [89,90]:

$$
\min _{W_{i}, h_{i}} \sum_{j=1}^{F}\left(\left\|x_{j}-W_{j}^{T} h_{j}\right\|^{2}+\lambda\left\|h_{j}\right\|_{1}\right)
$$

This is a convex objective in every $W_{i}$ and $h_{i}$ when the other is fixed. $\ell_{1}$ penalty term is the driving force in the above object forces for the sparse latent variable [88]. Here, the aforementioned objective is implemented for $W_{1}$ and $h_{1}$. Having sparse distributed representation (SDR) in this autoencoder not only follows the fundamental direction of deep learning but also creates robustness against noise [91]. Having our data compressed, we use a random forest to stratify the participants based on the sparse-latent deep features (Figure 3).

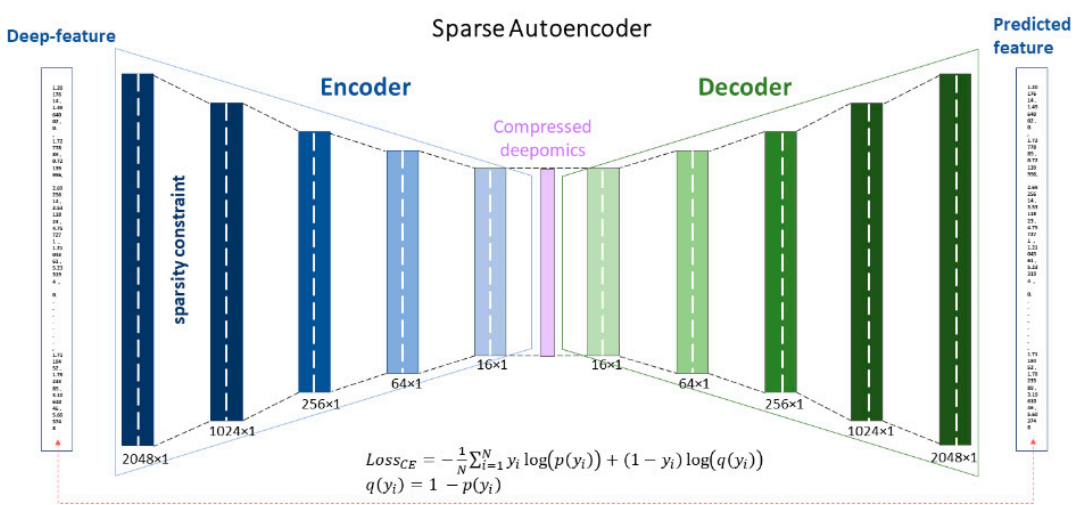

Figure 3. The proposed sparse deep autoencoder to reduce the dimensionality of the deep-thermomics is presented.

\section{Results}

The proposed method for thermal pattern detection was examined by thermal breast cancer screening datasets. The results of the low-rank approximation using sparse PCT were then compared to PCT thermal low-rank matrix approximation algorithm, as commonly used in infrared diagnostic systems. 


\subsection{Patient Population and Infrared Breast Cancer Study Data}

We used 208 participants from Database for Mastology Research (DMR) infrared breast screening dataset [92], who were healthy (without symptoms) or sick (diagnosed by mammographic imaging as breast cancer cases or non-cancerous but with symptoms). The median age in our study sample was 60 years, and the participants comprised 77 (37\%) Caucasian, 57 African (27.4\%), 72 Pardo (34.6\%), 1 Mulatto $(0.5 \%)$, and 1 indigenous (0.5\%) women. Among the participants, 52 had a history of diabetes in their families (25\%), and 38 were undergoing hormone replacement (18.3\%). All patients had infrared images obtained by the following acquisition protocol: images have a spatial resolution of $640 \times 480$ pixels and were captured by a FLIR thermal camera (model SC620) with a sensitivity of less than $0.04{ }^{\circ} \mathrm{C}$ range and capture standard of $-40{ }^{\circ} \mathrm{C}$ to $500{ }^{\circ} \mathrm{C}[27,92]$. Table 1 shows the clinical information and demography of the cohort. In this study, we considered symptomatic patients (who are not diagnosed with cancer but have similar signs) and sick (cancer) patients in one group, called the symptomatic group. The rationale behind this is due to having such analyses as the first line of screening, and once heterogeneity is detected by this system, further investigations need to be performed by a physician and another imaging modalities, i.e., mammography, to confirm the malignancy and specify the possible type of tumor.

Table 1. Clinical information and demographics of the breast cancer screening database using thermal imaging.

\begin{tabular}{|c|c|c|}
\hline \multicolumn{3}{|c|}{ DMR_Database for Mastology Research } \\
\hline Age & Median ( $( \pm \mathrm{IQR})$ & $60(25,120)$ \\
\hline \multirow{5}{*}{ Race } & Caucasian & $77(37 \%)$ \\
\hline & African & $57(27.4 \%)$ \\
\hline & Pardo & $72(34.6 \%)$ \\
\hline & Mulatto & $1(0.5 \%)$ \\
\hline & Indigenous & $1(0.5 \%)$ \\
\hline \multirow{3}{*}{ Diagnosis $^{1}$} & Healthy $^{2}$ & $128(61.5 \%)$ \\
\hline & Symptomatic (with and without cancer) & $80(38.5 \%)$ \\
\hline & Sick $^{3}$ & $36(17.3 \%)$ \\
\hline \multirow{4}{*}{ Family history } & Diabetes & $52(25 \%)$ \\
\hline & Hypertensive & $5(2.4 \%)$ \\
\hline & Leukemia & $1(0.5 \%)$ \\
\hline & None & $150(72.1 \%)$ \\
\hline \multirow{2}{*}{ Hormone therapy (HT) } & Hormone replacement & $38(18.3 \%)$ \\
\hline & None & $170(81.7 \%)$ \\
\hline
\end{tabular}

${ }^{1}$ This diagnosis performed with mammography as ground truth in this Dataset. ${ }^{2}$ Healthy term is used as non-cancerous and non-symptomatic patients. ${ }^{3}$ We use the term "sick", which includes different types of breast cancer patients diagnosed by mammographic imaging.

\subsection{Results of Low-Rank Sparse PCT (Principal Component Analysis)}

Three low-rank matrices were extracted from the 23 initially thermal sequences by using Sparse PCT. Some representative results of the low-rank approximation manually selected for our study cohort are shown in Figure 4. Low-rank approximation in the sequence of thermal images resulted in a heterogeneous breast area for 80 participants for breast cancer screening (sick and healthy with symptoms versus completely healthy without any symptom, Figure $4 \mathrm{a}-\mathrm{c}$ ). Thermal patterns showed more heterogeneous textures presenting the vasodilatory effect on the subdermal area of the breast. However, there was much less thermal heterogeneity found among the healthy participants (Table 1, Figure $4 \mathrm{~d}-\mathrm{f}$ ). The targeted areas indicate significantly lesser heterogeneous patterns projected by the low-rank Sparse PCA in the ROI. 


\subsection{Deep-Thermomic Features}

We extracted 2048 deep thermomic features from the targeted ROI in the thermal imaging (solely breasts area) using the latest layer of the ResNet-50 pre-trained model. The ResNet-50 pre-trained model contained five identical blocks having a convolutional layer, max-pooling, $\mathrm{ReLu}$, and many repetitive identity connections between each layer. Convolutional blocks consisted of three convolution layers like identity block. The ResNet-50 model had 25,583,592 trainable parameters and 53,120 non-trainable parameters. The preferred input image to ResNet-50 was an RGB image with a dimension of $224 \times 224 \times 3$. This squared image slipped through the entire process and ResNet-50 model re-scaled the spatial dimensions of the input image from 224 to 230, 112, 56, 28, 14, 7 while the fourth dimension grew from 3 to 64, 128, 256, 512, and 2048. Here, we used this to leverage the low-rank matrix approximation for each participant. We extracted three bases using sparse low-rank matrix approximations from the original thermal stream and stacked them like three channels in the input image. The input image was cropped around the ROI to create a square matrix for each channel identically.

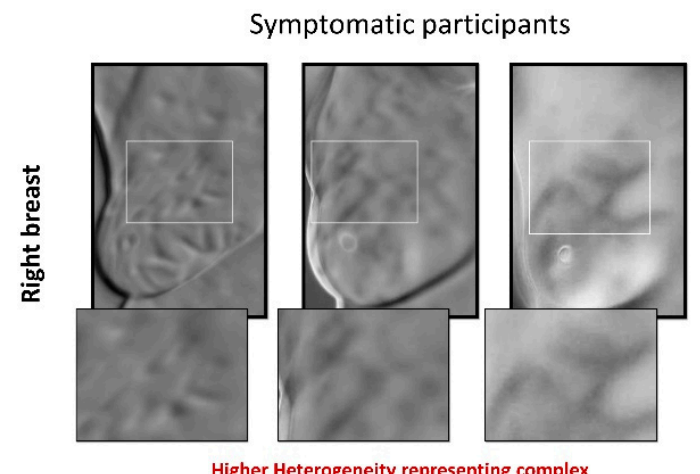

Higher Heterogeneity representing complex vasodilatory activity

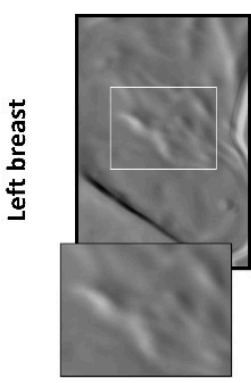

a.

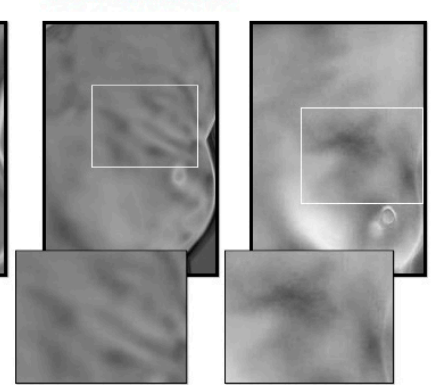

b.

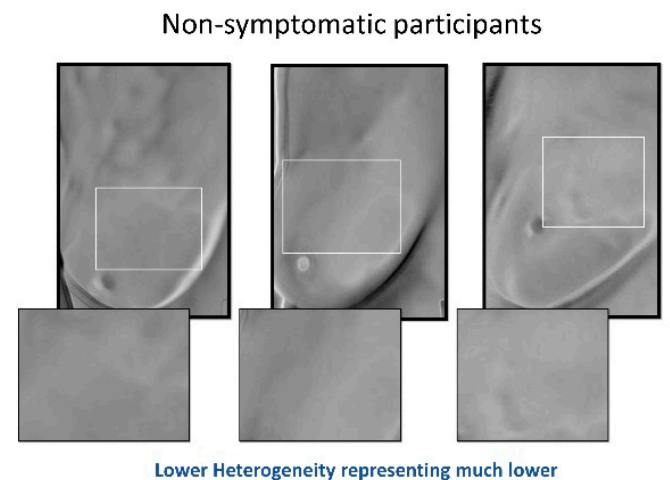

Lower Heterogeneity representing much lower

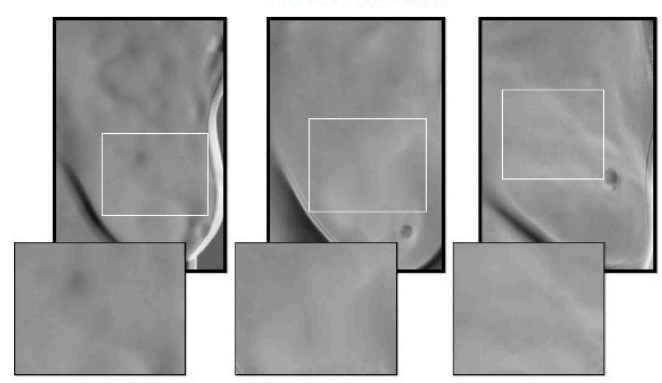

d.

e.

f.

Figure 4. Low-rank approximation of thermal sequence determined using different Sparse PCT (principal component analysis) matrix factorization technique. Each column shows different case, columns (a-c) show symptomatic patients (diagnosed by mammography as cancer patients or healthy with symptoms), whereas columns (d-f) show the result of methods for healthy cases.

\subsection{Result of the Sparse Autoencoder and Dimensionality Reduction}

We used 2048 deep features extracted from the ResNet-50 pre-trained model as the input of the proposed autoencoder. The autoencoder consisted of seven layers (Figure 3 ) and reduced the dimensionality from 2048 to 1024, 256, 64, and 16. The model was trained and validated with 4000, and 2000 vectors obtained by the ResNet-50 model from infrared images in the breast screening dataset. The model had 4,744,784 trainable parameters and was trained by Adam optimizer with the learning $\ell_{1}$ with a regularization value of $10^{-5}$ and for 500 epochs. The batch size was 128 for the model network. Figure 5 shows the loss of the model during the training.

From 2048 initially extracted deep thermomic features, the extracted features from the battle-neck layer of autoencoder resulted in 16 deep-thermomic features (Figure 5). Subsequently, the level of 
the heterogeneity for each participant measured through compressed descriptors was obtained by 128 times compression on the deep-thermomics.

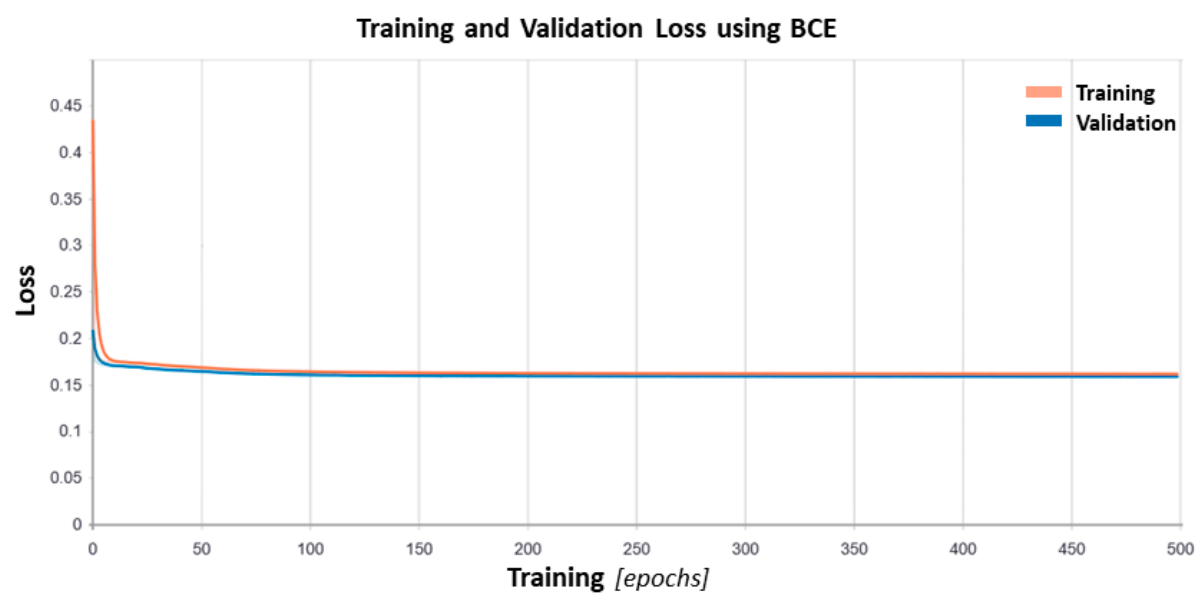

Figure 5. The binary cross entropy loss is presented for training and validation of the proposed sparse autoencoder for 300 epochs.

\subsection{Result of Random Forest Classification of Symptomatic and Non-Symptomatic Participants}

We stratified the participants based on the 16 sparse latent deep thermomic descriptors and compared them with the ground truth data based on mammography information. To examine the hypothesis that the thermal heterogeneity extracted by the deep learning model can be used as a biomarker to stratify among participants, a random forest classifier was fitted for multivariate covariates with leave-one-out cross-validation. The best multivariate model resulted accuracy of $75.24 \%(72.33-77.67 \%)$ for Sparse PCT, which was challenged by other matrix approximation technique PCT (73.27\% (71.84-76.21\%)). A multivariate model contains clinical information and demographics (age, and family history) gave an accuracy of $71.36 \%$ (69.42-73.3\%). A full multivariate model having all clinical and demographic information with the extracted features resulted in $78.16 \%$ (73.3-81.07\%) for Sparse PCT and $73.79 \%$ (72.33-76.7\%) for PCT (see Table 2). The receiver operating characteristic (ROC) curve of comparative analyses of baseline models is shown in Figure 6. The entire computational experiments were conducted by Python programming language [93] (for training and testing the model).

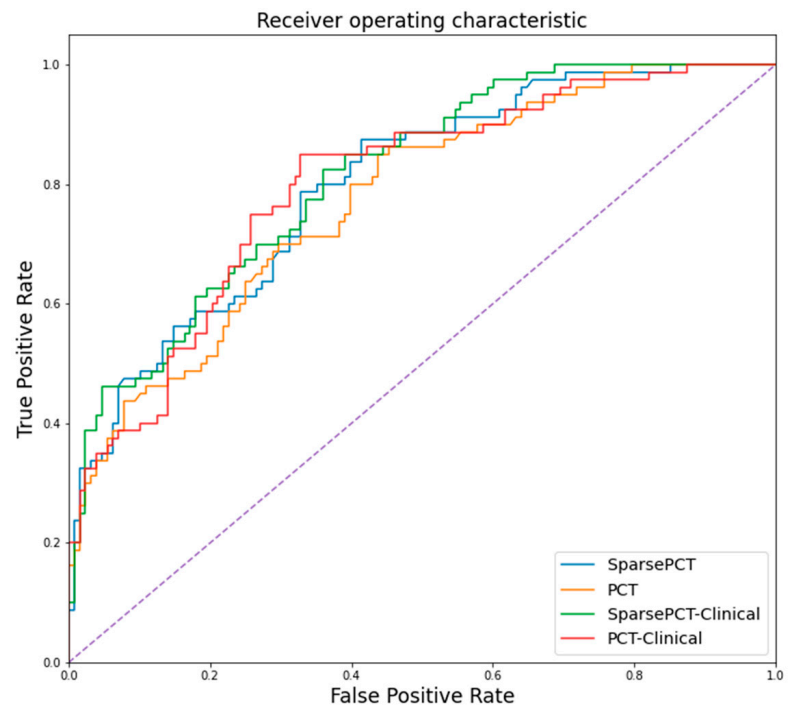

Figure 6. Receiver operating characteristic (ROC) curve for different multivariate model using deep thermomic features and clinical and demographic information is presented for classifying between symptomatic and non-symptomatic participants (for baseline model). 
Table 2. The results of random forest classification for the cross-validated model.

\begin{tabular}{ll}
\hline Methods & Cross-Validated Accuracy \\
\hline Sparse PCT & $75.24(72.33-77.67) \%$ \\
PCT & $73.27(71.84-76.21) \%$ \\
Clinical information * & $71.36(69.42-73.3) \%$ \\
Sparse PCT with clinical information * & $78.16(73.3-81.07) \%$ \\
PCT with clinical information * & $73.79(72.33-76.7) \%$ \\
\hline \multicolumn{2}{c}{${ }^{*}$ Clinical and demographic covariates: age, and family history. }
\end{tabular}

\section{Discussion}

In this study, we proposed a system to reduce the dimensionality of deep-thermomic features to extract thermal patterns for infrared diagnostic systems for thermography imaging. This study was designed based on the general trend of dimensionality reduction and to alleviate the possibility of over-fitting but used sparse multiple low-rank matrix approximations. This study showed a possibility to identify potential patients with breast cancer along with other clinical throughputs (such as CBE) using non-invasive, faster, and more cost-efficient thermography imaging.

The application of deep sparse autoencoder not only reduced the initial high-dimensional deep-thermomics but also added sparsity to the initial sparse representative of the thermal stream, which theoretically increases the robustness of the system against noise. It also showed significant improvements in stratifying symptomatic patients from healthy participants (Figure 6, and Table 2). Moreover, using sparse PCT showed higher accuracy than other approaches in finding heterogeneous thermal patterns, which might be due to the nature of sparsity in the calculation of the low-rank representative of the basis matrices, which were preserved by ResNet-50 level-level features and recursive training of the autoencoder network. This indicates the penalty terms in Sparse PCT creates constraints that worked in favor of detecting symptomatic cases while eliminating noise.

The application of deep thermomics considerably increased the dimensionality of the input thermal imaging and intensify the possibility of overfitting the random forest model, called the curse of dimensionality. The proposed sparse autoencoder reduced the dimensionality by removing the redundancy among the features by spanning thermomics to lower-dimensional space, while increasing the robustness of feature selection due to rigorous training of the model (this method is also used in other applications in medicine such as segmentation [94]). Since the infrared images used in this study had intensity information similar to natural images, using this pre-trained model is seemingly appropriate despite the medical nature of the analysis.

Thermal and infrared imagery has been used to determine breast abnormality, as the first medical application of thermography [92]. There are many discussions about more suitable positions for such imaging acquisitions [35] and the reliability of this modality $[57,58]$ that have been reported. However, the association of sparse autoencoder on the abundant deep thermomics finding thermal heterogeneity with a breast abnormality has not been discussed in literature which increases the novelty of this contribution to the field. One of the reasons that the proposed system performs well finding thermal heterogeneity might be because of the association of low-level deep features representing basis set and their sensitivity to slight intensity variation of thermal images. This can be justified by vasodilatory activity in the ROI for symptomatic patients. Despite some argument against using a thermal infrared imaging system as a solo-imaging modality for detecting breast abnormality, this technology has still been used as one of the important diagnostic tools with CBE and other imaging modalities (as discussed in Section 2).

One limitation to applying the presented models is related to data, and even with a considerable number of cases there is a need to increase the cohort to confirm the accuracy of the system. Having a larger cohort of patients increases the statistical power of such analysis by increasing the possibility of independently validating the system (substitute cross-validation). The other limitation may be using limited deep thermomic features. Having more deep-thermomics helps to assess the strength of 
the sparse autoencoder approach to select better compressing features that lead to capturing better thermal characteristics. There is an inherent limitation of using infrared thermography for detecting cancerous tissues once they are deeper into the tissues, as despite tracking the vasodilatory effects in the skin subsurface, deeper lesions might not be easy to detect. This might require further investigation using multimodal imaging analyses. There are also discussions on using a single thermal frame or multiple frames for selecting variability of thermal patterns across the acquisition time using different infrared-based approaches [5,35,95-97], which can be investigated further. Applying multiple thermal frames provided a chance of capturing thermal heterogeneity in the ROI for the duration of the acquisition, which might not be recorded by a single frame input system.

The presented technique offers some advantages. First, applying low-rank matrix approximation to extract thermal avatars during imaging acquisition provides a significant projection of thermal heterogeneity leading to better diagnosis of abnormal patients. Second, a sparse autoencoder eliminates the manual selection or human-engineering feature selection for reducing the dimensionality of the deep thermomics. Third, the proposed method considerably alleviates the effect of motion artifacts and imaging acquisition noise, which can be substantial improvements in infrared thermography applications. To the best of our knowledge, this is the first study which performs such analyses.

\section{Conclusions}

This study addressed one of the biggest challenges in high-dimensional deep feature selection, which selected the best representative deep thermomics from high-dimensional features extracted from a pre-trained deep neural networks model. The method performed multilayer dimensionality reduction using Sparse PCT to select the low-rank approximation of the thermal sequence. They extracted high-dimensional features from the ResNet-50 pre-trained model. Then, it used a trained sparse autoencoder to hierarchically reduce the size of the feature to 16 thermomic descriptors. We tested our method for 208 thermal breast cancer screening cases. We compared the appropriateness of these approaches with similar state-of-the-art thermographic methods, i.e., PCT. The results indicated the significant performance of the full multivariate model using Sparse PCT in preserving thermal heterogeneity to discriminate between symptomatic and healthy participants (accuracy of $78.16 \%$ (73.3-81.07\%)).

Future works should involve more thermomics extracted from the different low-rank approximations to increase the potential of assessing the entire thermal characteristics of cancerous parenchymal tissues. Moreover, an expansion of the validation set to a larger infrared imaging cohort can further confirm the strength and limitations of this approach.

Author Contributions: Conceptualization, B.Y. and H.A.; methodology, software, validation, formal analysis, investigation, resources, B.Y.; writing—original draft preparation, B.Y.; writing一review and editing, B.Y., H.A., X.P.V.M.; visualization, B.Y.; supervision, X.P.V.M. All authors have read and agreed to the published version of the manuscript.

Funding: This research received no external funding.

Acknowledgments: This research is conducted under a tier-one-Canadian Research Chair in Multipolar Infrared Vision (MIVIM) at the Department of Electrical and Computer Engineering at Laval University.

Conflicts of Interest: The authors declare no conflict of interest.

\section{References}

1. Siegel, R.L.; Miller, K.D.; Jemal, A. Cancer statistics, 2019. CA Cancer J. Clin. 2019, 69, 7-34. [CrossRef] [PubMed]

2. Crystal, P.; Strano, S.D.; Shcharynski, S.; Koretz, M.J. Using Sonography to Screen Women with Mammographically Dense Breasts. Am. J. Roentgenol. 2003, 181, 177-182. [CrossRef]

3. Kerlikowske, K.; Grady, D.; Barclay, J.; Sickles, E.A.; Ernster, V. Effect of age, breast density, and family history on the sensitivity of first screening mammography. JAMA 1996, 276, 33-38. [CrossRef] [PubMed] 
4. Hong, S.; Song, S.Y.; Park, B.; Suh, M.; Choi, K.S.; Jung, S.E.; Kim, M.J.; Lee, E.H.; Lee, C.W.; Jun, J.K. Effect of Digital Mammography for Breast Cancer Screening: A Comparative Study of More than 8 Million Korean Women. Radiology 2020, 294, 247-255. [CrossRef]

5. Kennedy, D.A.; Lee, T.; Seely, D. A comparative review of thermography as a breast cancer screening technique. Integr. Cancer Ther. 2009, 8, 9-16. [CrossRef]

6. Fletcher, S.W.; Elmore, J.G. Mammographic Screening for Breast Cancer. N. Engl. J. Med. 2003, 348, 1672-1680. [CrossRef] [PubMed]

7. Osako, T.; Iwase, T.; Takahashi, K.; Lijima, K.; Miyagi, Y.; Nishimura, S.; Tada, K.; Makita, M.; Akiyama, F.; Sakamoto, G.; et al. Diagnostic mammography and ultrasonography for palpable and nonpalpable breast cancer in women aged 30 to 39 years. Breast Cancer 2007, 14, 255-259. [CrossRef] [PubMed]

8. Duffy, S.W.; Vulkan, D.; Cuckle, H.; Parmar, D.; Sheikh, S.; Smith, R.A.; Evans, A.; Blyuss, O.; Johns, L.; Ellis, I.O.; et al. Effect of mammographic screening from age 40 years on breast cancer mortality (UK Age trial): Final results of a randomised, controlled trial. Lancet Oncol. 2020, 21, 1165-1172. [CrossRef]

9. Nguyen, Q.D.; Nguyen, N.T.; Dixon III, L.; Monetto, F.E.P.; Robinson, A.S. Spontaneously Disappearing Calcifications in the Breast: A Rare Instance Where a Decrease in Size on Mammogram Is Not Good. Cureus 2020, 12, e8753. [CrossRef]

10. De González, A.B.; Reeves, G. Mammographic screening before age 50 years in the UK: Comparison of the radiation risks with the mortality benefits. Br. J. Cancer 2005, 93, 590-596. [CrossRef] [PubMed]

11. Hoekstra, P. Quantitive digital thermology: 21st century imaging systems. In Proceedings of the OAND Conference, Hamilton, ON, Canada, 18-21 June 2001.

12. Law, J.; Faulkner, K.; Young, K.C. Risk factors for induction of breast cancer by X-rays and their implications for breast screening. Br. J. Radiol. 2007, 80, 261-266. [CrossRef] [PubMed]

13. Hendrick, R.E. Radiation Doses and Risks in Breast Screening. J. Breast Imaging 2020, 2, 188-200. [CrossRef]

14. Chowdhury, A.S.; Tamanna, S. Radiation-induced side effects in breast cancer patients and factors affecting them. Asian J. Med Biol. Res. 2020, 6, 138-148. [CrossRef]

15. Nelson, H.D.; Pappas, M.; Cantor, A.; Griffin, J.; Daeges, M.; Humphrey, L. Harms of breast cancer screening: Systematic review to update the 2009 US Preventive Services Task Force recommendation. Ann. Intern. Med. 2016, 164, 256-267. [CrossRef]

16. Bandyopadhyay, U.; Das, D.; Banerjee, R.K. Reactive oxygen species: Oxidative damage and pathogenesis. Curr. Sci. 1999, 77, 658-666.

17. Yang, Y.; Bazhin, A.V.; Werner, J.; Karakhanova, S. Reactive Oxygen Species in the Immune System. Int. Rev. Immunol. 2013, 32, 249-270. [CrossRef]

18. Friedenson, B. Is mammography indicated for women with defective BRCA genes? Implications of recent scientific advances for the diagnosis, treatment, and prevention of hereditary breast cancer. MedGenMed 2000, 2, E9. [PubMed]

19. Narod, S.A.; Lubinski, J.; Ghadirian, P.; Lynch, H.T.; Moller, P.; Foulkes, W.; Rosen, B.; Kim-Sing, C.; Isaacs, C.; Domcheck, S.; et al. Screening mammography and risk of breast cancer in BRCA1 and BRCA2 mutation carriers: A case-control study. Lancet Oncol. 2006, 7, 402-406. [CrossRef]

20. Khalkhali, I.; Vargas, H. Practical use of ultrasound at a dedicated breast center. Breast J. 2005, 11, $165-166$. [CrossRef]

21. Burkett, B.J.; Hanemann, C.W. A Review of Supplemental Screening Ultrasound for Breast Cancer. Acad. Radiol. 2016, 23, 1604-1609. [CrossRef]

22. Berg, W.A.; Zhang, Z.; Lehrer, D.; Jong, R.A.; Pisano, E.D.; Barr, R.G.; Böhm-Vélez, M.; Mahoney, M.C.; Evans, W.P.; Larsen, L.H.; et al. Detection of Breast Cancer With Addition of Annual Screening Ultrasound or a Single Screening MRI to Mammography in Women With Elevated Breast Cancer Risk. JAMA 2012, 307, 1394-1404. [CrossRef]

23. Morris, E.A. Screening for breast cancer with MRI. In Seminars in Ultrasound, CT and MRI. WB Saunders 2003, 24, 45-54.

24. Ibrahim, A.; Mohammed, S.; Ali, H.A. Breast Cancer Detection and Classification Using Thermography: A Review. In Proceedings of the International Conference on Advanced Machine Learning Technologies and Applications, Cairo, Egypt, 22-24 February 2018; Springer: Cham, Switzerland, 2018; pp. 496-505. 
25. Ahern, C.H.; Shih, Y.-C.T.; Dong, W.; Parmigiani, G.; Shen, Y. Cost-effectiveness of alternative strategies for integrating MRI into breast cancer screening for women at high risk. Br. J. Cancer 2014, 111, 1542-1551. [CrossRef]

26. Barton, M.B.; Harris, R.; Fletcher, S.W. Does This Patient Have Breast Cancer? JAMA 1999, 282, 1270-1280. [CrossRef]

27. Oestreicher, N.; Lehman, C.D.; Seger, D.J.; Buist, D.S.M.; White, E. The Incremental Contribution of Clinical Breast Examination to Invasive Cancer Detection in a Mammography Screening Program. Am. J. Roentgenol. 2005, 184, 428-432. [CrossRef]

28. Freed, D.; Golden, J.; Chu, M.; Carrillo, O.; Chin, Y.; Adams, M.; Abramov, V. Medical Devices and Systems. U.S. Patent Application No.11/388,247, 23 March 2006.

29. Kirimtat, A.; Krejcar, O.; Selamat, A.; Herrera-Viedma, E. FLIR vs SEEK thermal cameras in biomedicine: Comparative diagnosis through infrared thermography. BMC Bioinform. 2020, 21, 1-10. [CrossRef] [PubMed]

30. Moore, G. Breast cancer: Early detection needed. Bus. Heal. 2001, 19, 39.

31. Feig, S.A.; Shaber, G.S.; Schwartz, G.F.; Patchefsky, A.; Libshitz, H.I.; Edeiken, J.; Nerlinger, R.; Curley, R.F.; Wallace, J.D. Thermography, Mammography, and Clinical Examination in Breast Cancer Screening. Radiology 1977, 122, 123-127. [CrossRef]

32. Keyserlingk, J.; Ahlgren, P.; Yu, E.; Belliveau, N.; Yassa, M. Functional infrared imaging of the breast. IEEE Eng. Med. Boil. Mag. 2000, 19, 30-41. [CrossRef] [PubMed]

33. Anbar, M. Clinical thermal imaging today. IEEE Eng. Med. Boil. Mag. 1998, 17, 25-33. [CrossRef] [PubMed]

34. Gautherie, M. Thermopathology of breast cancer: Measurement and analysis of in vivo temperature and blood flow. Ann. N. Y. Acad. Sci. 1980, 335, 383-415. [CrossRef]

35. Recinella, A.N.; Gonzalez-Hernandez, J.-L.; Kandlikar, S.G.; Dabydeen, D.; Medeiros, L.; Phatak, P. Clinical Infrared Imaging in the Prone Position for Breast Cancer Screening-Initial Screening and Digital Model Validation. J. Eng. Sci. Med. Diagn. Ther. 2020, 3. [CrossRef]

36. Mc Donald, D. Mechanism of Tumour Leakiness Proceeding Angiogenesis and Cancer. From Basic Mechanisms to Therapeutic Applications. In Proceedings of the American Association of Cancer Research Conference (AACR), San Francisco, CA, USA, 1-5 April 2000; pp. 11-15.

37. Gamagami, P. Indirect Signs of Breast Cancer: Angiogenesis Study. In Atlas of Mammography; Blackwell Science: Cambridge, MA, USA, 1996; pp. 321-326.

38. Yoshida, S.; Nakagawa, S.; Yahara, T.; Koga, T.; Deguchi, H.; Shirouzu, K. Relationship Between Microvessel Density and Thermographic Hot Areas in Breast Cancer. Surg. Today 2003, 33, 243-248. [CrossRef]

39. Mitra, S.; Bal, A.; Kashyap, D.; Kumar, S.; Shrivastav, S.; Das, A.; Singh, G. Tumour angiogenesis and c-Met pathway activation-Implications in breast cancer. APMIS 2020, 128, 316-325. [CrossRef]

40. Tsutsui, S.; Yasuda, K.; Suzuki, K.; Tahara, K.; Higashi, H.; Era, S. Macrophage infiltration and its prognostic implications in breast cancer: The relationship with VEGF expression and microvessel density. Oncol. Rep. 2005, 14, 425-431. [CrossRef]

41. Buckley, D.L.; Drew, P.J.; Mussurakis, S.; Monson, J.R.; Horsman, A. Microvessel density in invasive breast cancer assessed by dynamic gd-dtpa enhanced MRI. J. Magn. Reson. Imaging 1997, 7, 461-464. [CrossRef]

42. Anbar, M.; Brown, C.; Milescu, L.; Babalola, J.; Gentner, L. The potential of dynamic area telethermometry in assessing breast cancer. IEEE Eng. Med. Boil. Mag. 2000, 19, 58-62. [CrossRef] [PubMed]

43. Thomsen, L.L.; Miles, D.W.; Happerfield, L.; Bobrow, L.G.; Knowles, R.G.; Moncada, S. Nitric oxide synthase activity in human breast cancer. Br. J. Cancer 1995, 72, 41-44. [CrossRef]

44. Arnedos, M.; Vicier, C.; Loi, S.; Lefebvre, C.; Michiels, S.; Bonnefoi, H.; Andre, F. Precision medicine for metastatic breast cancer-Limitations and solutions. Nat. Rev. Clin. Oncol. 2015, 12, 693. [CrossRef]

45. Di Meo, S.; Reed, T.T.; Venditti, P.; Victor, V.M. Role of ROS and RNS Sources in Physiological and Pathological Conditions. Oxidative Med. Cell. Longev. 2016, 2016, 1-44. [CrossRef]

46. Moldogazieva, N.T.; Lutsenko, S.V.; Terentiev, A.A. Reactive Oxygen and Nitrogen Species-Induced Protein Modifications: Implication in Carcinogenesis and Anticancer Therapy. Cancer Res. 2018, 78, 6040-6047. [CrossRef] [PubMed]

47. Smith, K.A.; Waypa, G.B.; Schumacker, P.T. Redox signaling during hypoxia in mammalian cells. Redox Biol. 2017, 13, 228-234. [CrossRef]

48. Xu, W.; Liu, L.Z.; Loizidou, M.; Ahmed, M.J.U.; Charles, I.G. The role of nitric oxide in cancer. Cell Res. 2002, 12, 311-320. [CrossRef] 
49. Choudhari, S.K.; Chaudhary, M.; Bagde, S.; Gadbail, A.R.; Joshi, V. Nitric oxide and cancer: A review. World J. Surg. Oncol. 2013, 11, 1-11. [CrossRef]

50. Martínez, M.C.; Andriantsitohaina, R. Reactive Nitrogen Species: Molecular Mechanisms and Potential Significance in Health and Disease. Antioxidants Redox Signal. 2009, 11, 669-702. [CrossRef]

51. Xing, F.; Liu, Y.; Sharma, S.; Wu, K.; Chan, M.D.; Lo, H.-W.; Carpenter, R.L.; Metheny-Barlow, L.J.; Zhou, X.; Qasem, S.A.; et al. Activation of the c-Met Pathway Mobilizes an Inflammatory Network in the Brain Microenvironment to Promote Brain Metastasis of Breast Cancer. Cancer Res. 2016, 76, 4970-4980. [CrossRef]

52. Changchun, K.; Pengchao, H.; Ke, S.; Ying, W.; Wei, L. Interleukin-17 augments tumor necrosis factor $\alpha$-mediated increase of hypoxia-inducible factor- $1 \alpha$ and inhibits vasodilator-stimulated phosphoprotein expression to reduce the adhesion of breast cancer cells. Oncol. Lett. 2017, 13, 3253-3260. [CrossRef]

53. Maher, E.A.; Mietz, J.; Arteaga, C.L.; DePinho, R.A.; Mohla, S. Brain metastasis: Opportunities in basic and translational research. Cancer Res. 2009, 69, 6015-6020. [CrossRef]

54. Ganong, W.F. Review of Medical Physiology, 2nd ed.; Lange Medical Books/McGraw-Hill: San Francisco, CA, USA, 2005.

55. Spychala, J.; Lazarowski, E.; Ostapkowicz, A.; Ayscue, L.H.; Jin, A.; Mitchell, B.S. Role of estrogen receptor in the regulation of ecto-5'-nucleotidase and adenosine in breast cancer. Clin. Cancer Res. 2004, 10, 708-717. [CrossRef]

56. Lozano, A.; Hayes, J.C.; Compton, L.M.; Azarnoosh, J.; Hassanipour, F. Determining the thermal characteristics of breast cancer based on high-resolution infrared imaging, 3D breast scans, and magnetic resonance imaging. Sci. Rep. 2020, 10, 1-14. [CrossRef] [PubMed]

57. FDA Safety Communication, FDA Warns Thermography Should Not Be Used in Place of Mammography to Detect, Diagnose, or Screen for Breast Cancer. 2019. Available online: www.fda.gov (accessed on 25 February 2019).

58. FDA Consumer Update, Breast Cancer Screening: Thermogram No Substitute for Mammogram, U.S. Food and Drug Administration. 2017. Available online: www.fda.gov (accessed on 30 October 2017).

59. Pennes, H.H. Analysis of Tissue and Arterial Blood Temperatures in the Resting Human Forearm. J. Appl. Physiol. 1948, 1, 93-122. [CrossRef]

60. Rajic, N. Principal component thermography for flaw contrast enhancement and flaw depth characterisation in composite structures. Compos. Struct. 2002, 58, 521-528. [CrossRef]

61. Yousefi, B.; Sfarra, S.; Ibarra-Castanedo, C.; Maldague, X.P. Comparative analysis on thermal non-destructive testing imagery applying Candid Covariance-Free Incremental Principal Component Thermography (CCIPCT). Infrared Phys. Technol. 2017, 85, 163-169. [CrossRef]

62. Yousefi, B.; Sfarra, S.; Sarasini, F.; Castanedo, C.I.; Maldague, X.P. Low-rank sparse principal component thermography (sparse-PCT): Comparative assessment on detection of subsurface defects. Infrared Phys. Technol. 2019, 98, 278-284. [CrossRef]

63. Wu, J.-Y.; Sfarra, S.; Yao, Y. Sparse Principal Component Thermography for Subsurface Defect Detection in Composite Products. IEEE Trans. Ind. Inform. 2018, 14, 5594-5600. [CrossRef]

64. Usamentiaga, R.; Mokhtari, Y.; Ibarra-Castanedo, C.; Klein, M.; Genest, M.; Maldague, X. Automated Dynamic Inspection Using Active Infrared Thermography. IEEE Trans. Ind. Inform. 2018, 14, 5648-5657. [CrossRef]

65. Marinetti, S.; Finesso, L.; Marsilio, E. Matrix factorization methods: Application to thermal NDT/E. NDT E Int. 2006, 39, 611-616. [CrossRef]

66. Cramer, K.E.; Winfree, W. Fixed eigenvector analysis of thermographic NDE data. SPIE 2011, $225-235$. [CrossRef]

67. Yousefi, B.; Sharifipour, H.M.; Eskandari, M.; Castanedo, C.I.; Laurendeau, D.; Watts, R.; Klein, M.; Maldague, X. Incremental Low Rank Noise Reduction for Robust Infrared Tracking of Body Temperature during Medical Imaging. Electronics 2019, 8, 1301. [CrossRef]

68. Ahmed, J.; Gao, B.; Woo, W.L. Wavelet-Integrated Alternating Sparse Dictionary Matrix Decomposition in Thermal Imaging CFRP Defect Detection. IEEE Trans. Ind. Inform. 2018, 15, 4033-4043. [CrossRef]

69. Zou, H.; Hastie, T.; Tibshirani, R. Sparse principal component analysis. J. Comput. Graph. Stat. 2006, 15, 265-286. [CrossRef]

70. FeCun, Y. LeNet-5, Convolutional Neural Networks. 2015. Available online: http://yann.lecun.com/exdb/lenet (accessed on 31 October 2020). 
71. Aghdam, H.H.; Heravi, E.J. Guide to Convolutional Neural Networks: A Practical Application to Traffic-Sign Detection and Classification; Springer: Berlin/Heidelberg, Germany, 2017.

72. Krizhevsky, A.; Sutskever, I.; Hinton, G.E. ImageNet classification with deep convolutional neural networks. In Proceedings of the Advances in Neural Information Processing Systems (NIPS), Lake Tahoe, NV, USA, 3-6 December 2012; pp. 1097-1105.

73. He, K.; Zhang, X.; Ren, S.; Sun, J. Deep Residual Learning for Image Recognition. In Proceedings of the 2016 IEEE Conference on Computer Vision and Pattern Recognition (CVPR), Las Vegas, NV, USA, 26 June-1 July 2016; pp. 770-778.

74. Simonyan, K.; Zisserman, A. Very deep convolutional networks for large-scale image recognition. arXiv 2014, arXiv:1409.1556.

75. Szegedy, C.; Liu, W.; Jia, Y.; Sermanet, P.; Reed, S.; Anguelov, D.; Erhan, D.; Vanhoucke, V.; Rabinovich, A. Going deeper with convolutions. In Proceedings of the 2015 IEEE Conference on Computer Vision and Pattern Recognition (CVPR), Boston, MA, USA, 7-12 June 2015; pp. 1-9.

76. He, K.; Zhang, X.; Ren, S.; Sun, J. Identity Mappings in Deep Residual Networks. arXiv 2016, arXiv:1603.05027v3.

77. Liang, L.; Liu, M.; Sun, W. A deep learning approach to estimate chemically-treated collagenous tissue nonlinear anisotropic stress-strain responses from microscopy images. Acta Biomater. 2017, 63, 227-235. [CrossRef]

78. Akbari, H.; Rathore, S.; Bakas, S.; Nasrallah, M.P.; Shukla, G.; Mamourian, E.; Rozycki, M.; Bagley, S.J.; Rudie, J.D.; Flanders, A.E.; et al. Histopathology-validated machine learning radiographic biomarker for noninvasive discrimination between true progression and pseudo-progression in glioblastoma. Cancer 2020, 126, 2625-2636. [CrossRef]

79. Yousefi, B.; Kalhor, D.; Usamentiaga, R.; Lei, L.; Ibarra-Castanedo, C.; Maldague, X. Application of Deep Learning in Infrared Non-Destructive Testing. Quant. InfraRed Thermogr. 2018. [CrossRef]

80. Chaves, E.; Gonçalves, C.B.; Albertini, M.K.; Lee, S.; Jeon, G.; Fernandes, H.C. Evaluation of transfer learning of pre-trained CNNs applied to breast cancer detection on infrared images. Appl. Opt. 2020, 59, E23-E28. [CrossRef]

81. Acharya, S.; Alsadoon, A.; Prasad, P.W.C.; Abdullah, S.; Deva, A. Deep convolutional network for breast cancer classification: Enhanced loss function (ELF). J. Supercomput. 2020, 76, 1-18. [CrossRef]

82. Fernández-Ovies, F.J.; Alférez-Baquero, E.S.; De Andrés-Galiana, E.J.; Cernea, A.; Fernández-Muñiz, Z.; Fernández-Martínez, J.L. Detection of Breast Cancer Using Infrared Thermography and Deep Neural Networks. In Proceedings of the International Work-Conference on Bioinformatics and Biomedical Engineering, Granada, Spain, 8-10 May 2019; Springer: Cham, Switzerland, 2019; pp. 514-523.

83. Zuluaga-Gomez, J.; Al Masry, Z.; Benaggoune, K.; Meraghni, S.; Zerhouni, N. A CNN-based methodology for breast cancer diagnosis using thermal images. Comput. Methods Biomech. Biomed. Eng. Imaging Vis. 2020, 1-15. [CrossRef]

84. Ekici, S.; Jawzal, H. Breast cancer diagnosis using thermography and convolutional neural networks. Med. Hypotheses 2020, 137, 109542. [CrossRef]

85. Mambou, S.J.; Maresova, P.; Krejcar, O.; Selamat, A.; Kuca, K. Breast Cancer Detection Using Infrared Thermal Imaging and a Deep Learning Model. Sensors 2018, 18, 2799. [CrossRef]

86. He, K.; Zhang, X.; Ren, S.; Sun, J. Deep Residual Learning for Image Recognition. arXiv 2015, arXiv:1512.03385.

87. Gillies, R.J.; Kinahan, P.E.; Hricak, H. Radiomics: Images Are More than Pictures, They Are Data. Radiology 2016, 278, 563-577. [CrossRef]

88. Chollet, F. Building Autoencoders in Keras. 2016. Available online: https://blog.keras.io/buildingautoencoders-in-keras.html (accessed on 14 March 2017).

89. Olshausen, B.A.; Field, D.J. Sparse coding with an overcomplete basis set: A strategy employed by V1? Vis. Res. 1997, 37, 3311-3325. [CrossRef]

90. Arpit, D.; Zhou, Y.; Ngo, H.; Govindaraju, V. Why regularized auto-encoders learn sparse representation? In Proceedings of the International Conference on Machine Learning, New York, NY, USA, 19-24 June 2016; pp. 136-144.

91. Hinton, G.E. Distributed Representations. 1984. Available online: https://web.stanford.edu/ \{\}\}lmcc/papers/ PDP/ (accessed on 18 July 2005). 
92. Silva, L.F.; Saade, D.C.M.; Sequeiros, G.O.; Silva, A.C.; Paiva, A.C.; Bravo, R.S.; Conci, A. A New Database for Breast Research with Infrared Image. J. Med Imaging Heal. Inform. 2014, 4, 92-100. [CrossRef]

93. Python 3 Google Compute Engine Backend, T4, and P100 GPU and 27.4 Gb RAM, Google 2020.

94. Mortazi, A.; Khosravan, N.; Torigian, E.A.; Kurugol, S.; Bagci, U. Weakly Supervised Segmentation by a Deep Geodesic Prior. In Lecture Notes in Computer Science; Springer: Cham, Switzerland, 2019; pp. 238-246.

95. Yousefi, B.; Castanedo, C.I.; Maldague, X.P. Measuring heterogeneous thermal patterns in infrared-based diagnostic systems using sparse low-rank matrix approximation: Comparative study. IEEE Trans. Instrum. Meas. 2020. [CrossRef]

96. Yousefi, B.; Castanedo, C.I.; Maldague, X.P.V. Low-rank Convex/Sparse Thermal Matrix Approximation for Infrared-based Diagnostic System. arXiv 2020, arXiv:2010.06784.

97. Kirimtat, A.; Krejcar, O. A review of infrared thermography for the investigation of building envelopes: Advances and prospects. Energy Build. 2018, 176, 390-406. [CrossRef]

Publisher's Note: MDPI stays neutral with regard to jurisdictional claims in published maps and institutional affiliations.

(C) 2020 by the authors. Licensee MDPI, Basel, Switzerland. This article is an open access article distributed under the terms and conditions of the Creative Commons Attribution (CC BY) license (http://creativecommons.org/licenses/by/4.0/). 